\title{
Determination of mechanical properties of historical paper based on NIR spectroscopy and chemometrics - a new instrument
}

\author{
Dirk Lichtblau ${ }^{1}$, Matija Strlič*2 ${ }^{2}$, Tanja Trafela ${ }^{3}$, Jana Kolar ${ }^{4}$, Manfred Anders ${ }^{1}$ \\ ${ }^{1}$ Zentrum für Bucherhaltung, Mommsenstrasse 7, 04329 Leipzig, Germany \\ ${ }^{2}$ University College London, Centre for Sustainable Heritage, The Bartlett School of \\ Graduate Studies (Torrington Place site), Gower Street, London WCIE 6BT, UK \\ ${ }^{3}$ University of Ljubljana, Faculty of Chemistry and Chemical Technology, Aškerčeva 5, \\ SI-1000 Ljubljana, Slovenia \\ ${ }^{4}$ National and University Library, Turjaška 1, SI-1000 Ljubljana, Slovenia \\ *e-mail: m.strlic@ucl.ac.uk
}

\begin{abstract}
Due to sampling restrictions in analysis of cultural heritage materials, non-destructive approaches are intensively sought for. While NIR spectrometry has rarely been used for this purpose due to the complexity of spectra, chemometric methods can be used to extract the necessary information. For the purpose of determination of mechanical properties of historical paper, partial least squares approach was used and it is shown that tensile strength and tensile strength after folding can be estimated based on NIR spectra. As mechanical properties of paper-based objects define their accessibility, a new dispersive portable instrument was built, which will enable us to rapidly survey the condition of library and archival collections.
\end{abstract}

\section{Keywords}

Analysis, near infrared spectroscopy, paper, conservation

\section{PACS}

82.80.Gk, 28.52.Fa, 28.52.Lf 


\section{Introduction}

In collections of paper-based cultural heritage, especially libraries and archives, mechanical properties of paper are the quality parameter defining its usability. Items, which are too fragile to be manipulated, may be considered inaccessible and the information severely endangered.

During natural ageing, loss of paper strength is the consequence of degradation processes of its main structural component, cellulose. Degree of polymerisation of cellulose has been shown to correlate with retention of folding endurance of a bleached sulphite pulp sample [1]. A review on the subject of mechanical properties during ageing was published by Gurnagul et al. [2].

Cellulose degradation proceeds according to several reaction pathways, two of which are especially important. Acid-catalysed hydrolysis prevails in cellulose in an acidic environment, while autoxidation becomes more important in cellulose in neutral and mildly alkaline environment $[3,4]$. Historically, acids in paper are a consequence of rosin sizing, which became widespread in papermaking around ca. 1850 and discontinued due to environmental concerns around 1990. Prior to ca. 1850, gelatine sizing was used, which rendered $\mathrm{pH}$ of paper surface slightly acidic [5]. Despite this, historical gelatinesized paper is generally still in a good condition if it has been kept in favourable environmental conditions, in the absence of corrosive inks or biodeteriogens. In fact, it has been shown that gelatine is beneficial to paper and increases its longevity [6]. Today, we are faced with kilometres of shelves of highly unstable acidic paper in many libraries and archives. In surveys of these collections, the usability of paper is sometimes tested using the manual fold test [7], which is not well repeatable and certainly destructive. There are standardised methods for determination of mechanical properties of paper, e.g. tensile strength (ISO 1924-2:1994), tearing resistance (ISO 1974:1990), bursting strength (ISO 2758:2001), folding endurance (ISO 5626:1993) etc., however, they require a large sample and many repetitions, since the measurements exhibit low repeatability. In any case, neither of the two approaches is suitable for historical objects, as the samples are damaged during analysis. Clearly, another approach is called for. Since mechanical properties of paper are defined by both intra- and intermolecular and interfibre bonds in the material [8], it is feasible that with spectroscopic methods we 
might be able to assess their number and identity. Due to the variety of possible bonds, a chemometric approach is called for, with which the mechanical properties could be correlated with spectral information. Additionally, NIR spectra are dominated by overtones and combination bands, and contain fewer specific features than mid-IR spectra. To extract the necessary information, chemometric analysis of data is a widespread approach instead of band assignation [9] and partial least-squares (PLS) allows for correlation of spectral and chemical information [10-12]. However, the quality of these correlations depends on a number of factors, among which the quality of spectra and the quality of analytical data play a decisive role. The use of partial least squares (PLS) approach for this purpose was first reported in [13] and was recently applied to determination of several chemical parameters of historical paper using FT-NIR and midIR [14]. It was even shown that dating can be attempted.

In this paper, we demonstrate that mechanical properties of historical paper can also be determined using NIR/mid-IR spectra and PLS calibration, and we report on development of an instrument, with which rapid determination of these properties may be possible in a library or archival environment. In addition, this approach is essentially multi-analytical and reagent-free. It could easily be used for other materials, and it is believed that it will find widespread use in conservation laboratories.

\section{Experimental}

Paper samples (49) were taken from books dated from 1870 till present day, randomly collected through acquisition or donations. A wide variety of paper types was used, containing different pulps, from fully bleached to groundwood, and different sizes, from rosin-sized to contemporary paper. Paper fibre composition, determined according to SCAN-G3:90, and publication year are shown in Table 1.

Parts with evident damage due to biodegradation, water-related damage or with damage evidently caused by excessive use were disregarded. Excessively soiled and densely printed samples or laminated, transparent and other specialty papers were not considered either. Margins $(3 \mathrm{~cm})$ were cut away to exclude areas degraded due to environmental influences. 
Table 1: Samples used in the study along with publication year, data on fibre composition and tensile strength, both actually measured and predicted using NIR/PLS.

\begin{tabular}{|c|c|c|c|c|c|c|c|c|}
\hline \multirow[b]{2}{*}{ No. } & \multirow[b]{2}{*}{ Year } & \multicolumn{3}{|c|}{ fibre composition } & \multicolumn{2}{|c|}{ tensile strength $(\mathrm{N})$} & \multicolumn{2}{|c|}{ tensile strength after folding $(\mathrm{N})$} \\
\hline & & groundwood & $\begin{array}{l}\text { bleached } \\
\text { pulp }\end{array}$ & cotton & measured & predicted & measured & predicted \\
\hline 1 & 1871 & & 0.10 & 0.90 & 58.9 & 58.2 & 20.6 & 23.9 \\
\hline 2 & 1899 & & 1.00 & & 58.9 & 60.7 & 21.6 & 20.0 \\
\hline 3 & 1910 & 0.20 & 0.80 & & 31.7 & 32.0 & 19.6 & 16.6 \\
\hline 4 & 1910 & 0.20 & 0.80 & & 73.0 & 75.0 & 16.3 & 16.0 \\
\hline 5 & 1911 & & 1.00 & & 49.4 & 42.3 & 24.2 & 20.1 \\
\hline 6 & 1913 & 0.10 & 0.10 & 0.80 & 42.3 & 41.6 & 18.6 & 18.7 \\
\hline 7 & 1917 & 0.30 & 0.70 & & 22.8 & 23.3 & 23.5 & 22.7 \\
\hline 8 & 1921 & 0.40 & 0.60 & & 53.2 & 52.3 & 24.9 & 22.7 \\
\hline 9 & 1922 & 0.50 & 0.50 & & 53.2 & 50.2 & 16.6 & 21.0 \\
\hline 10 & 1922 & 0.40 & 0.60 & & 42.3 & 43.8 & 14.7 & 18.4 \\
\hline 11 & 1928 & 0.50 & 0.50 & & 31.0 & 29.0 & 41.2 & 42.2 \\
\hline 12 & 1928 & 0.60 & 0.40 & & 31.7 & 40.1 & 26.8 & 21.7 \\
\hline 13 & 1932 & 0.70 & 0.30 & & 31.0 & 32.2 & 22.3 & 19.8 \\
\hline 14 & 1933 & 0.80 & 0.20 & & 37.1 & 35.3 & 35.1 & 30.4 \\
\hline 15 & 1934 & 0.60 & 0.40 & & 29.3 & 36.4 & 28.1 & 28.1 \\
\hline 16 & 1935 & & & 1.00 & 29.3 & 29.4 & 35.9 & 34.4 \\
\hline 17 & 1936 & & 1.00 & & 73.0 & 68.6 & 27.1 & 30.6 \\
\hline 18 & 1938 & 0.10 & 0.90 & & 45.8 & 44.5 & 36.4 & 33.6 \\
\hline 19 & 1938 & 0.80 & 0.20 & & 37.1 & 38.4 & 26.5 & 25.6 \\
\hline 20 & 1938 & 0.70 & 0.30 & & 29.2 & 32.3 & 27.4 & 24.9 \\
\hline 21 & 1939 & & 0.10 & 0.90 & 57.4 & 59.7 & 39.8 & 36.7 \\
\hline 22 & 1939 & 0.90 & 0.10 & & 63.2 & 62.2 & 32.3 & 35.5 \\
\hline 23 & 1939 & 0.05 & 0.95 & & 63.2 & 62.2 & 27.1 & 32.2 \\
\hline 24 & 1940 & 0.85 & 0.15 & & 45.8 & 47.9 & 20.1 & 20.3 \\
\hline 25 & 1940 & 0.65 & 0.35 & & 59.9 & 60.3 & 33.0 & 34.0 \\
\hline 26 & 1940 & & 0.70 & 0.30 & 45.0 & 46.4 & 21.3 & 19.7 \\
\hline 27 & 1942 & 0.55 & 0.45 & & 48.9 & 50.7 & 27.3 & 29.1 \\
\hline 28 & 1943 & 0.90 & 0.10 & & 64.5 & 66.0 & 21.8 & 25.9 \\
\hline 29 & 1943 & 0.85 & 0.15 & & 59.9 & 59.5 & 23.7 & 24.4 \\
\hline 30 & 1944 & 0.80 & 0.20 & & 45.0 & 45.8 & 39.4 & 38.8 \\
\hline 31 & 1945 & 0.10 & 0.90 & & 57.4 & 53.5 & 37.4 & 39.8 \\
\hline 32 & 1945 & 0.30 & 0.70 & & 29.2 & 32.1 & 21.5 & 17.1 \\
\hline 33 & 1958 & 0.60 & 0.40 & & 64.5 & 61.4 & 11.6 & 11.7 \\
\hline 34 & 1958 & & 1.00 & & 22.8 & 27.5 & 26.5 & 30.6 \\
\hline 35 & 1965 & & 1.00 & & 49.4 & 47.4 & 20.0 & 24.3 \\
\hline 36 & 1966 & & 1.00 & & 63.6 & 64.9 & 46.0 & 43.2 \\
\hline 37 & 1972 & & 0.70 & 0.30 & 40.4 & 38.1 & 43.6 & 42.8 \\
\hline 38 & 1976 & & 1.00 & & 37.4 & 34.3 & 39.4 & 38.3 \\
\hline 39 & 1995 & & 1.00 & & 48.9 & 49.9 & 56.4 & 56.4 \\
\hline 40 & 1995 & & 1.00 & & 40.4 & 36.1 & 46.1 & 46.0 \\
\hline 41 & 1996 & & 1.00 & & 52.9 & 44.8 & 60.8 & 52.8 \\
\hline 42 & 1996 & & 1.00 & & 55.3 & 56.2 & 52.9 & 52.3 \\
\hline 43 & 2001 & & 1.00 & & 55.3 & 58.5 & 51.9 & 53.6 \\
\hline 44 & 1870 & & & 1.00 & 65.6 & 67.9 & 17.7 & 17.1 \\
\hline 45 & 1905 & & 1.00 & & 63.6 & 59.7 & 23.2 & 19.2 \\
\hline 46 & 1920 & & 1.00 & & 40.4 & 40.4 & 20.0 & 25.4 \\
\hline 47 & 1927 & 0.70 & 0.30 & & 52.9 & 47.0 & 24.5 & 25.9 \\
\hline 48 & 1957 & & 1.00 & & 40.4 & 40.2 & 19.3 & 22.2 \\
\hline 49 & 1957 & & 0.10 & 0.9 & 65.6 & 64.8 & 20.9 & 26.6 \\
\hline
\end{tabular}

For determination of tensile strength (ISO 1924-2:1994), we used Zwick Proline z0.5 TS instrument (Ulm, Germany), load cell nominal force $500 \mathrm{~N}$ type II, pneumatic grips with 
convex aluminium and steel jaw faces, jaw pressure 6 bar. The dimensions of paper stripes were $100 \times 15 \mathrm{~mm}$.

Tensile strength after folding was performed according to [15]. In order to fold paper stripes in a standardised manner, two ends are fixed together on a plane with $20^{\circ}$ inclination (Feinmechanik Ralf Kögel, Leipzig, Germany) and a cylinder of $460 \mathrm{~g}$ is triggered to roll down. The tensile strength of the folded sample was measured in the same way as without folding.

Regardless of the fact that tensile strength after folding is not a standardised method, the two techniques were chosen because they closely resemble the manual fold test as used in [7] and in many subsequent condition surveys in libraries and archives: a corner of a page is folded and pressed between two fingers as many times as it takes to break off the corner.

The mechanical properties were measured in the machine direction and the average uncertainty of the measured values was $2 \%$. The low uncertainty was also one of the reasons why determination of folding endurance was not our method of choice. The measured data are provided in Table 1.

A Perkin-Elmer Spectrum GX (Waltham, MA) equipped with a 76-mm Labsphere RSAPE-200-ID (North Sutton, NH) integration sphere coated with Infragold, with a DTGS detector, was used. The reflectance spectra were collected in the interval $6500-500 \mathrm{~cm}^{-1}$, 128 scans per sample. Spectra were taken using 4 layers of sample paper, as justified in [14].

Three independent sets of spectra were taken on three different positions on the same sample. Two sets were used for calibration and one for validation. Spectrum Quant+ software (Perkin Elmer, Waltham, MA), partial least squares analysis (PLS) was used to model the chemical properties. Optimal correlations were sought for, only by using different pretreatments of spectra and different wavenumber intervals. The predicted tensile strength data are given in Table 1.

To evaluate the quality of calibration, two standard errors were calculated [16]. The standard error of estimate (SEE) is the average error between the measured property values and the estimated values of the calibrated standards from a given model. SEE can be described as the square root of the residual variance divided by the number of degrees 
of freedom. The standard error of prediction (SEP) is the average error between the measured and estimated property values of samples not used in the calibration. SEP gives an estimate of the magnitude of the error expected when independent samples are predicted using the developed model.

\section{Results and discussion}

Different pre-treatments of spectra (smoothing, derivation) and different wavenumber intervals were tested until optimal PLS calibrations were obtained and model parameters are given in Table 2 . The sole criterion for selecting the best pre-treatment and wavenumber interval was the quality of PLS validations. Derivation of spectra gave the best results and the wavenumber intervals used are provided in Table 2 . The spectra used for these calculations were obtained using the Perkin-Elmer Spectrum GX equipped with an integration sphere.

For validation, a separate set of spectra was used. The calibration and validation models are given in Figure 2. Considering the variability of samples (Table 1), typical of real collections, the correlations are satisfactory. It is of interest that good quality correlations are obtained without having to take into account dimensional properties, e.g. paper thickness.

Table 2. Summary of the parameters of PLS calibrations and validations for different mechanical properties $(N$ - number of spectra, $S E E$ - standard error of estimation, $S E P$ - standard error of prediction).

\begin{tabular}{|l|c|c|c|c|c|}
\hline Mechanical & Wavenumber & \multicolumn{3}{|c|}{ Calibration } & Validation \\
\cline { 3 - 6 } property & interval $\left(\mathrm{cm}^{-1}\right)$ & $N$ & SEE & SEP & $N$ \\
\hline Tensile strength & $6500-1000$ & 98 & 3,391 & 3,557 & 49 \\
\hline $\begin{array}{l}\text { Tensile strength } \\
\text { after folding }\end{array}$ & $6500-1000$ & 98 & 3,524 & 3,627 & 49 \\
\hline
\end{tabular}



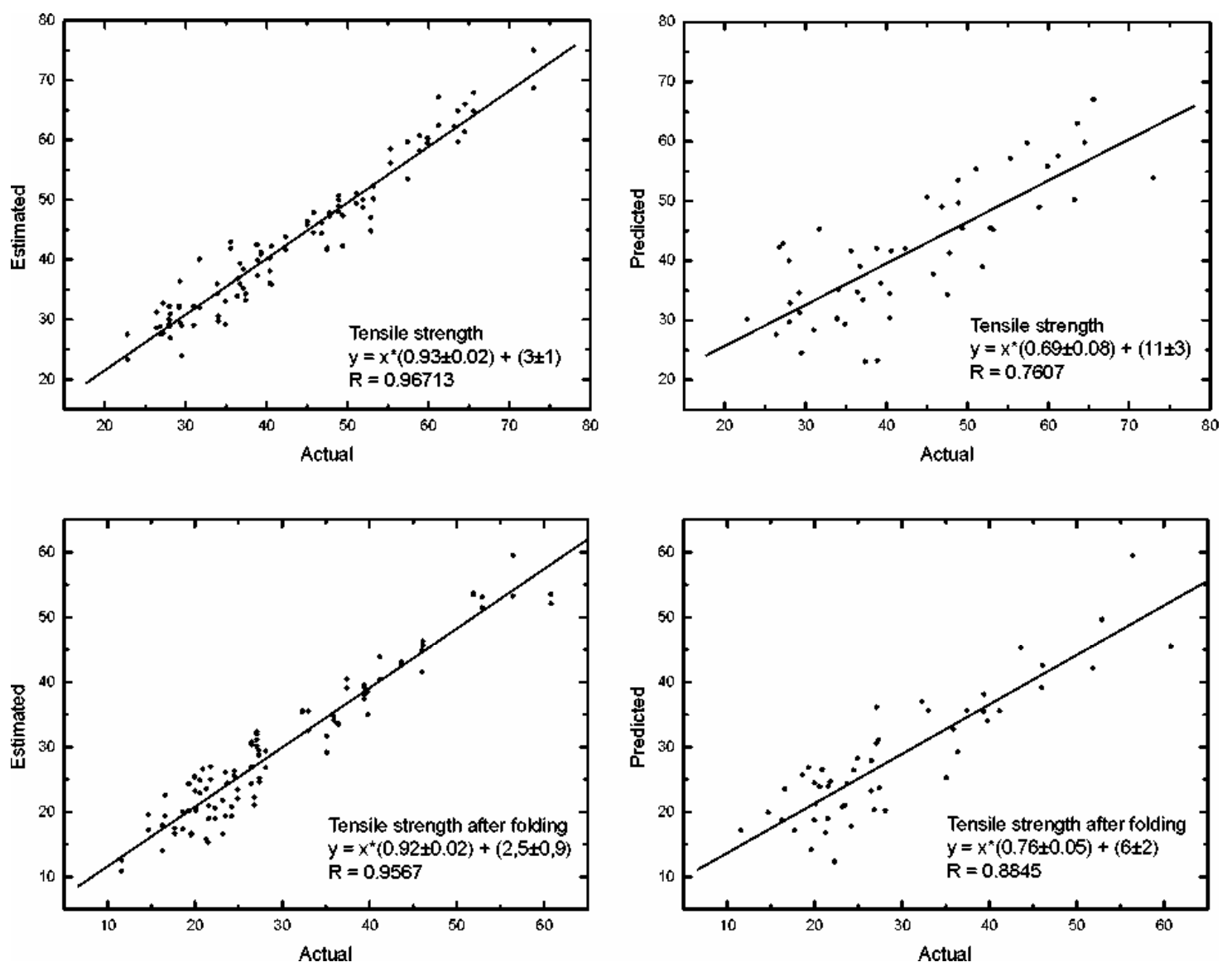

Figure 1. PLS calibrations (left) and validations (right) for estimation and prediction of selected mechanical properties of historical paper.

In evaluation of the quality of correlations, we have to take into account the uncertainty of determination of mechanical properties of paper, which is bigger than that of chemical properties [14]. It is to be expected that the uncertainty is even higher due to sample inhomogeneity brought about by ageing. Although the uncertainty of predictions is higher than the uncertainty of real data, i.e. determinations of mechanical properties, the demonstrated approach is very useful for a rapid and non-destructive estimation of whether the object in question is mechanically stable enough for regular use or not, without destructive testing.

Additionally, based on such measurements, appropriate conservation procedures can be undertaken. If the object in question can withstand manipulation (and possibly swelling in hydrophilic solvents) without loss of historical substance due to its low mechanical stability, it can undergo stabilization treatments using solvents, such as deacidification, 
which is used to neutralize acids in paper and supply an alkaline reserve to protect cellulose during future degradation. If the mechanical strength does not allow such a treatment, other types of conservation or preservation actions can be considered, including reformatting.

However, in order to fully exploit the potential of the developed approach, the Fouriertransform NIR/mid-IR instrument used for the above method development is not practical for everyday use in conservation laboratories, as it is a bench-top instrument and whole items such as historical books or documents cannot be examined using the integration sphere. Thus, an instrument had to be developed to satisfy the need for portability and versatility in terms of sample size. In addition, such an instrument had to provide maximum security for the measured object and ease of operation.

In the frame of the SurveNIR project [17], a dispersive instrument prototype was built of the size $350 \times 240 \times 150 \mathrm{~mm}$ and weight less than $10 \mathrm{~kg}$. The instrument makes use of the wavelength interval $1100-2500 \mathrm{~nm}\left(9100-4000 \mathrm{~cm}^{-1}\right.$, respectively). This interval was chosen for several reasons: it encompasses most of the NIR region while the size and the weight of the developed instrument could be minimized in order to allow for portability. A measurement is done in the way as shown in Figure 2, which is safe for the object and allows us to adapt to object size easily. The measured object is kept at a safe distance from the 2-mm measurement aperture and the use of a smooth Perspex table minimizes the possibilities of damage to the object. An automatic shutter system reduces the illumination time to $<1 \mathrm{~s}$, which is enough to record several hundreds of spectra and calculate an average spectrum. 


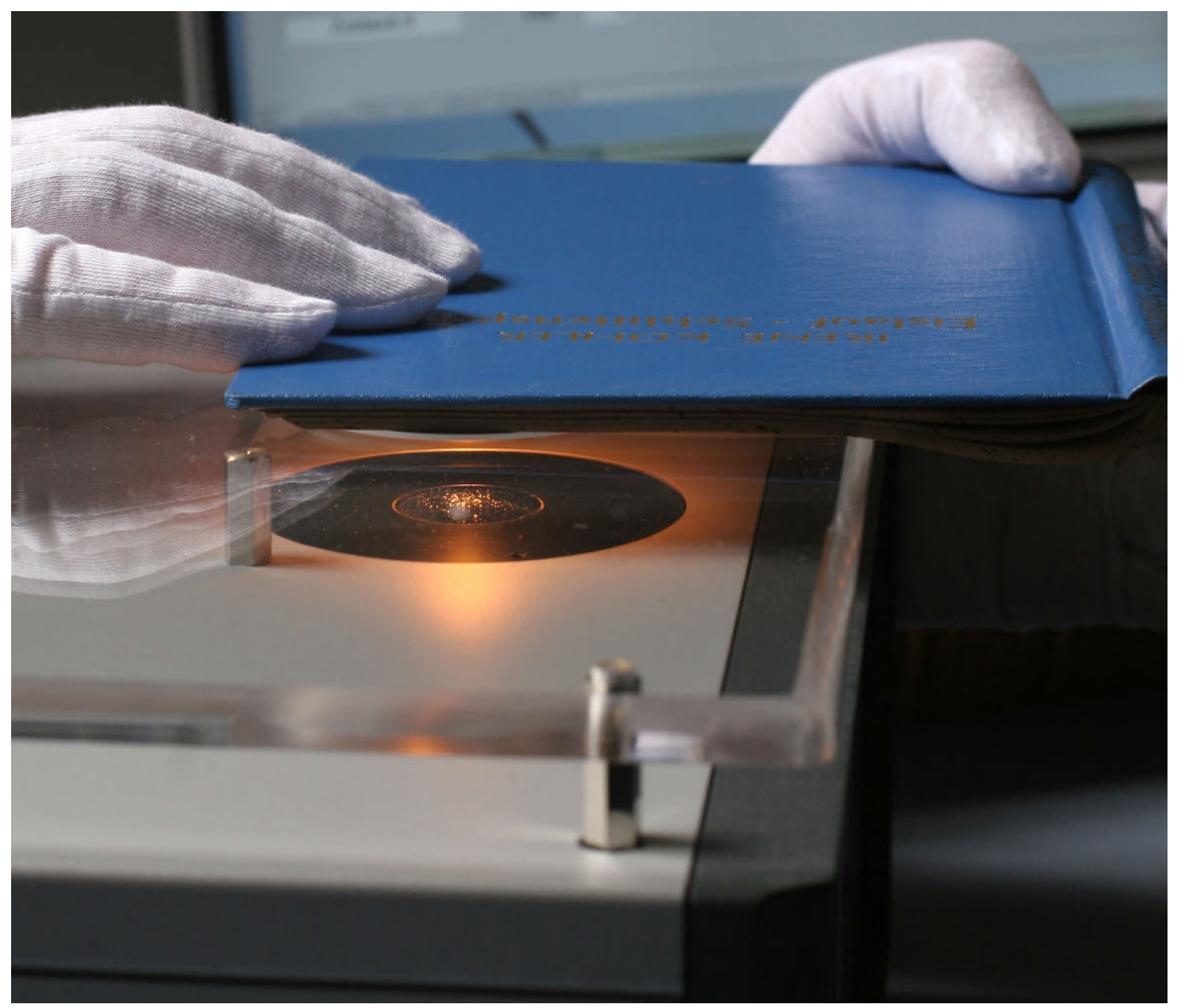

Figure 2. The SurveNIR instrument prototype. The table made of Perspex is positioned at a fixed distance from the measurement aperture, so that there is no direct contact between an item and the measurement opening.

The prototype was purpose-built for conducting surveys of large paper-based collections as it allows rapid object handling and data acquisition. During such surveys, the condition of a whole collection can be assessed in order to plan for large-scale preservation and conservation actions. Traditionally, surveys are performed using subjective tests, such as the manual folding test, however, it is well known that subjectivity seriously influences the results of such surveys [18]. The instrumental approach is expected to both accelerate the surveying procedures and increase their reliability. The prototype is in the testing phase and our current research focuses on development and optimization of PLS models for this instrument. 


\section{Conclusions}

Partial least squares approach was used to correlate NIR spectral information with mechanical properties of historical paper. It was shown that tensile strength and tensile strength after folding can be successfully predicted from NIR spectra. This enables us to determine mechanical properties in a non-destructive manner. Based on the obtained information, appropriate conservation actions can be undertaken.

A portable dispersive NIR instrument was built in order to fully exploit the potential of the technique for examination of paper-based objects in library and archival situations.

\section{Acknowledgements}

The authors gratefully acknowledge the support of the European Community, 6th Framework Energy, Environment and Sustainable Development programme, contract no. SSPI-006594 (SurveNIR). The work is the sole responsibility of authors and does not represent the opinion of the Community. The Community is not responsible for any use that might be made of the data appearing herein.

Further financial support has been obtained from the Ministry of Higher Education, Science and Technology of Slovenia, programme no. P1-0153.

\section{References}

1 X. Zou, T. Uesaka, N. Gurnagul: Cellulose 3, 243 (1996)

2 N. Gurnagul, R.C. Howard, X. Zou, T. Uesaka, D.H. Page: J. Pulp Pap. Sci. 19, J160 (1993)

3 J. Kolar: Restaurator 18, 163 (1997)

4 M. Strlič, J. Kolar: Ageing and Stabilisation of Paper (National and University Library, Ljubljana 2005)

5 A. L. Dupont: Gelatine Sizing of Paper and Its Impact on the Degradation of Cellulose during Ageing (Ph.D. Dissertation, University of Amsterdam, 2003.

6 M. Strlič, J. Kolar, D. Kočar, T. Drnovšek, V.-S. Šelih, R. Susič, B. Pihlar: ePreserv. Sci. 1, 35 (2004)

$7 \quad$ S. Buchanan, S. Coleman: Coll. Res. Libr. 48, 102 (1987) 
8 J. C. Roberts: The Chemistry of Paper (The Royal Society of Chemistry, Cambridge 1996)

9 M. Blanco, I. Villarroya: TrAC, Trends Anal. Chem. 240, 21 (2002)

10 G. R. Brereton: Chemometrics, Data Analysis for the Laboratory and Chemical Plant (Wiley \& Sons, Chichester 2003)

11 H. P. Garthwaite: J. Am. Stat. Assoc. 112, 89, 1994

12 A. Höskuldsson: Chemom. Intell. Lab. Syst. 23, 55, 2001

13 M. Anders, P. Behnsen, P. Plew: Prepr. X. IADA Congr., Göttingen, Germany, p. $11(2003)$

14 T. Trafela, M. Strlič, J. Kolar, D. A. Lichtblau, M. Anders, D. Pucko Mencigar, B. Pihlar: Anal. Chem. Anal. Chem. 79, 6319 (2007)

15 H. Bansa, H. H. Hofer: Papier 34, 348 (1980)

16 H. W. Siesler, Y. Ozaki, S. Kawata, H. M. Heise: Near-Infrared Spectroscopy. Principles, instruments, Applications (Wiley-VCH, Weinheim 2002)

17 www.science4heritage.org/survenir (accessed 14/06/2007)

18 J. Taylor, S. Stevenson: Mus. Manag. Curator. 18, 19 (1999). 